\title{
Perdas de oportunidades na prevenção do câncer de colo uterino durante o pré-natal
}

\author{
Missed opportunities \\ for cervical cancer prevention during prenatal care
}

Carla Vitola Gonçalves ${ }^{1}$

Geraldo Duarte ${ }^{2}$

Juvenal Soares Dias da Costa ${ }^{3}$

Silvana Maria Quintana ${ }^{2}$

Alessandra Cristina Marcolin ${ }^{2}$

${ }^{1}$ Departamento MaternoInfantil, Faculdade de Medicina, Fundação Universidade Federal do Rio Grande. Av. Visconde de Paranaguá 102, Centro. 96020-550 Rio Grande RS. carlavg@brturbo.com.br ${ }^{2}$ Departamento MaternoInfantil, Faculdade de Medicina de Ribeirão Preto, Universidade de São Paulo. ${ }^{3}$ Departamento de Medicina Social, Faculdade de Medicina, Universidade Federal de Pelotas.
Abstract Pregnancy constitutes an excellent opportunity for the prevention of cervical carcinoma since the gynecological examination is part of routine prenatal care. A transversal study was conducted in which a total of 445 postnatal women were interviewed using standardized questionnaires. The prevalence of an up-to-date cytopathological exam was $38.9 \%$ at the beginning of pregnancy, reaching $59.1 \%$ during the postnatal period $(p>0.001)$. Postnatal women aged 19 years or less, non-white, with less than 11 years schooling, family income of less than one minimum wage, sexually active at 15 years of age or less, with the beginning of prenatal care after the 1st trimester, and receiving prenatal care at healthcare units of the Unified Health System had a lower prevalence of cytopathological examination. Adjusted analysis revealed that the variables under study were not significantly associated with cytopathological coverage, though the incidence of prenatal care showed a prevalence ratio of 1.18 (95\% CI: 0.98 1.42). The local health service proved ineffective, recvealing the need to increase cytopathological coverage and train health professionals regarding the importance of routine prenatal procedures.

Key words Prenatal care, Prevention of cervical cancer, Assessing the quality of healthcare, Health inequalities
Resumo A gravidez representa uma excelente oportunidade para prevenção do carcinoma cervical, já que o exame ginecológico faz parte da rotina de pré-natal. Foi realizado um estudo transversal, no qual foram entrevistadas 445 puérperas utilizando-se questionários padronizados. A prevalência de CP atualizado era de 38,9\% no início da gestação, chegando a 59,1\% no puerpério ( $p>0,001)$. As puérperas com 19 anos ou menos, não brancas, com escolaridade inferior a onze anos, com renda familiar inferior a um salário mínimo, sexarca aos 15 anos ou menos, início do pré-natal após o primeiro trimestre e com o acompanhamento no Sistema Único de Saúde (SUS) apresentaram menor cobertura do citopatológico. Na análise ajustada, essas variáveis não mostraram significância associada à cobertura do citopatológico. Entretanto, a realização do pré-natal mostrou uma tendência à melhora da cobertura do CP com razão de prevalência de 1,18 (95\%CI: 0,98-1,42). O serviço local de saúde mostrou-se pouco efetivo, revelando a necessidade de aumentar a cobertura do citopatológico, motivando e capacitando os profissionais quanto à importância dos procedimentos da rotina pré-natal.

Palavras-chave Assistência pré-natal, Prevenção do câncer do colo uterino, Avaliação da qualidade dos cuidados de saúde, Desigualdades em saúde 


\section{Introdução}

O câncer do colo uterino é a segunda neoplasia mais frequente nas mulheres em todo o mundo. Para o ano de 2007, a Organização Mundial da Saúde (OMS) estimou que 260.000 mulheres faleceriam dessa enfermidade no mundo, destacando que cerca de $95 \%$ dos casos ocorreriam nos países em desenvolvimento ${ }^{1}$.

No Brasil, em 2005, o câncer do colo uterino foi a terceira neoplasia maligna mais comum entre as mulheres $(19 / 100.000)^{2}$. Nesse mesmo ano, o carcinoma cervical foi a quarta causa de morte por câncer em mulheres, apresentando coeficiente de mortalidade de 4,8/100.000. Para 2008, o Instituto Nacional de Câncer (Inca) estima uma incidência de 18.680 casos novos de câncer do colo uterino no país.

No Estado do Rio Grande do Sul, a estimativa de câncer do colo uterino é de 1.610 casos novos para o ano de 2008, gerando uma taxa bruta de incidência de 31 casos/100.000 mulheres, sendo superado apenas pelo câncer de mama. O carcinoma cervical representa a quinta causa de morte por neoplasia no sexo feminino neste estado, apresentando um coeficiente de mortalidade de 5,9/100.000 mulheres no ano de $2005^{3}$.

Nos países em que a mortalidade por câncer de colo uterino se mantém elevada, a maioria das mulheres que desenvolveram esta neoplasia não realizou o exame citopatológico ou o fizeram com periodicidade inadequada, observando-se também inadequações técnicas na coleta e análise do material. Além desses problemas, observa-se tratamento inadequado em alguns casos ${ }^{4}$.

A OMS estima que $95 \%$ das mulheres que vivem em países classificados como "em desenvolvimento" nunca tenham se submetido ao exame citopatológico do colo uterino. Além disso, $80 \%$ das mulheres com diagnóstico recente de carcinoma cervical vivem em países em desenvolvimento, e em quase todas elas a enfermidade se encontra em estádios avançados ${ }^{1}$. No Brasil, com base em dados coletados em 96 centros oncológicos, entre 1995 e 2002, observou-se que $45,5 \%$ das pacientes com câncer de colo uterino encontravam-se nos estádios III ou IV no momento do diagnóstico inicial ${ }^{5}$.

As evidências atuais indicam que as gestantes apresentam chance três vezes maior de serem diagnosticadas como portadoras de lesões em estádio inicial do câncer de colo do que os controles, visto que nesse período os exames vaginais são mais frequentes. Como a maioria dessas lesões é assintomática, seu diagnóstico quase sempre ocor- re em consultas de controle, mais frequentes durante o pré-natal ${ }^{6,7}$. Alguns estudos sobre câncer de colo uterino durante a gravidez mostram que, no momento do diagnóstico, $70 \%$ a $80 \%$ das gestantes apresentam lesões no estádio I, enquanto fora da gravidez apenas $42 \%$ dos diagnósticos são realizados neste estádio ${ }^{7,8}$. Assim, a gravidez representa uma excelente oportunidade para prevenção do câncer do colo uterino, já que faz parte da rotina de pré-natal preconizada pela OMS e pelo Ministério da Saúde do Brasil. Faz parte dessa rotina a inspeção do colo uterino, a coleta de exame citopatólogico (quando o último exame tiver sido realizado há 36 meses ou mais) e a palpação bimanual ${ }^{6,79}$. Esses cuidados são enfatizados, visto que a atenção pré-natal pode ser o único contato que uma mulher em idade reprodutiva tem com o serviço de saúde d,9-12. $^{12}$.

No Brasil, observa-se que a cobertura numérica da assistência pré-natal, por si só, não garante bons resultados ${ }^{12}$. Dados referentes a 2006 demonstram uma cobertura pré-natal de $90 \%$ no país e 95,3\% no Estado do Rio Grande do $\mathrm{Sul}^{13}$. Apesar da alta cobertura pré-natal encontrada, observam-se desigualdades nos cuidados oferecidos às grávidas ${ }^{14}$. Segundo dados do Ministério da Saúde do Brasil, somente pequena parcela das gestantes inscritas nos programas de pré-natal conseguem realizar o elenco mínimo de ações preconizadas, demonstrando o comprometimento da qualidade da atenção pré-natal prestada neste país ${ }^{9,10}$. Essa situação traduz a inefetividade dos serviços de saúde no sentido de garantirem atendimento adequado não apenas em relação ao número de consultas, mas também ao conteúdo do atendimento oferecido ${ }^{12,15}$.

Os estudos que visam avaliar a qualidade dos serviços de saúde prestados em nosso país são escassos. A própria literatura especializada tem privilegiado a análise das características e dos resultados da assistência pelo número de consultas prénatais e tipo de parto, relegando a plano secundário o estudo da qualidade do conteúdo das consultas, embora esse tipo de avaliação retrate com maior fidelidade o processo do atendimento ${ }^{14,15}$.

Visto que o câncer de colo uterino é uma das neoplasias mais frequentes durante a vida reprodutiva, é de lógica irreparável aproveitar a consulta pré-natal para o diagnóstico de suas lesões precursoras. Se isso não for possível, que pelo menos se faça o diagnóstico do câncer em uma fase mais precoce. No entanto, o que se vê na prática é que a oportunidade de fazer essa avaliação no pré-natal não está sendo aproveitada na sua totalidade. 
Com este estudo, objetivou-se avaliar a cobertura do exame citopatológico do colo uterino durante o pré-natal e descrever características associadas ao não cumprimento dessa norma. Esses resultados possibilitarão instrumentalizar os gestores e autoridades da saúde do nosso país para o embasamento científico das intervenções necessárias para corrigir essa distorção.

\section{Materiais e métodos}

O estudo foi uma avaliação transversal, no qual foram aferidos: (1) a prevalência de gestantes com exame citopatológico do colo uterino atualizado (realizado nos últimos 36 meses); (2) a frequência de exame citopatológico realizado durante o pré-natal (caso este fosse indicado) e os fatores associados à não realização deste exame. Foi utilizado o delineamento transversal, por ser este o modelo mais adequado para avaliar programas de saúde, além de apresentar baixo custo e ser de rápida realização. O projeto foi aprovado pelo Comitê de Ética em Pesquisa da Fundação Universidade Federal do Rio Grande (FURG-RS).

Este estudo foi realizado no município do Rio Grande, localizado na planície costeira sul do Estado do Rio Grande do Sul, com uma área de 3.338 quilômetros quadrados. Sua população estimada em 2007 foi de 198.560 habitantes, sendo que 62.797 são mulheres em idade fértil ${ }^{13}$. O sistema de saúde do município conta com apenas dois hospitais, a Associação de Caridade Santa Casa do Rio Grande e o Hospital Universitário Dr. Miguel Riet Corrêa Jr. (HU-FURG), com capacidade total de 600 leitos, sendo que 56 são disponibilizados para obstetrícia, com média anual de 2.500 partos $^{13}$.

O tamanho da amostra foi calculado pelo Programa Epi-Info utilizando os seguintes parâmetros: população feminina em idade fértil estimada na cidade de Rio Grande em 2007, prevalência das gestantes com exame citopatológico do colo uterino atualizado (55\%) e erro amostral de 5\%, totalizando 378 puérperas com nível de confiança de $95 \%$.

Foram realizados 473 partos na Associação de Caridade Santa Casa do Rio Grande e no Hospital Universitário Dr. Miguel Riet Corrêa Jr. (FURG), de maio de 2007 a julho de 2007. O primeiro critério de inclusão foi que a finalização da gestação tivesse ocorrido após as 20 semanas, e o segundo foi a anuência da paciente (por escrito) em participar da pesquisa. Foram excluídas pacientes que não haviam realizado pelo menos uma consulta de pré-natal.
Das 473 puérperas, nove $(1,9 \%)$ pacientes se recusaram a realizar a entrevista, e 19 (4,0\%) não haviam realizado nenhuma consulta pré-natal, sendo excluídas da investigação. Ao final do trabalho de campo, foram realizadas 445 entrevistas, sendo 259 (58,2\%) no Hospital Universitário (FURG) e 186 (41,8\%) na Associação de Caridade Santa Casa do Rio Grande.

O grupo de entrevistadores foi composto por acadêmicos do curso de Medicina da FURG, treinados para a aplicação do questionário estruturado proposto inicialmente, sendo realizado estudo piloto. Após fazer as adaptações que o estudo piloto indicou, o questionário estruturado e pré-codificado foi aplicado às puérperas ainda durante a internação hospitalar, não ultrapassando um período de 48 horas após o parto. Esse instrumento continha variáveis demográficas, socioeconômicas, dados referentes ao pré-natal da gravidez atual, dados epidemiológicos que poderiam caracterizar fatores de risco para câncer do colo uterino e questionamentos sobre a cobertura do exame citopatológico do colo uterino antes e durante o pré-natal. Entre as variáveis referentes à qualidade do pré-natal, temos o índice de Kessner ${ }^{16}$, que foi considerado adequado quando as gestantes iniciavam o pré-natal no primeiro trimestre e realizavam seis ou mais consultas; inadequado quando a primeira consulta ocorreu no segundo ou terceiro trimestre e o número de consultas era igual ou inferior a cinco.

Após o término do trabalho de campo foi realizada a codificação dos dados, seguida de sua digitação no programa Epi-Info 6.04. A análise estatística utilizou as ferramentas do aplicativo SPSS, prevendo a utilização de análise bruta e ajustada, razão de prevalência, intervalos de confiança e p-valor.

A análise multivariada foi realizada através da Regressão de Poisson, pela possibilidade de demonstrar a magnitude das associações sem problemas de estimativa, oriundas de razões de diferenças. No Programa Stata, foi seguido um modelo hierarquizado de análise ${ }^{17}$, sendo que ingressaram as variáveis com teste no valor de até 0,20 na análise bruta. No primeiro nível, ingressaram as variáveis demográficas e socioeconômicas. No segundo, ingressaram no modelo as variáveis que representavam os antecedentes obstétricos e os fatores de risco para câncer de colo uterino. No terceiro nível, estavam as características do pré-natal atual. Em cada nível do modelo foram retiradas as variáveis que não obtiveram p-valor menor que 0,05 . 


\section{Resultados}

Entre as 445 puérperas incluídas no estudo, 424 $(95,3 \%)$ referiam conhecer o exame preventivo do câncer do colo uterino. Mesmo assim, apenas 252 pacientes $(56,6 \%)$ relatavam a realização do exame citopatológico do colo do útero $(\mathrm{CP}) \mathrm{em}$ algum momento de suas vidas. As outras 193 mulheres $(43,4 \%)$ referiram nunca terem sido submetidas ao exame de CP na vida.

Das 252 mulheres que apresentavam citologia cervical prévia no início do pré-natal, 173 $(68,7 \%)$ tinham realizado o exame de CP há menos de 36 meses e 79 (31,3\%) estavam com o exame desatualizado. Portanto, somando-se as 193 mulheres que referiram nunca terem se submetido ao exame de $\mathrm{CP}$ às 79 pacientes com citologia cervical realizada com mais de 36 meses, obteve-se um total de 272 gestantes $(61,1 \%)$ com o exame citopatológico não atualizado no início do pré-natal.

Os médicos pré-natalistas ofereceram o exame de CP a $111(40,8 \%)$ das 272 gestantes com citologia cervical não atualizada. No entanto, a coleta do CP no pré-natal foi realizada apenas em $90(81,1 \%)$ das pacientes em que esta foi oportunizada. Entre as 21 gestantes $(18,9 \%)$ que não realizaram o exame de CP no pré-natal, os motivos referidos foram: nove pacientes relataram medo de realizar a coleta por estarem grávidas, outras nove puérperas referiram dificuldades para agendar o exame e posteriormente terem que voltar à unidade de saúde para realizá-lo, duas apresentavam leucorreia no momento da coleta e uma paciente teve medo de sentir dor durante a realização do exame.

No final do pré-natal, a prevalência do exame citopatológico atualizado entre as puérperas foi de $59,1 \%$, sendo que 160 (36\%) das pacientes permaneceram sem nunca terem coletado o exame de citologia cervical e 22 puérperas $(4,9 \%)$ continuaram com o exame citopatológico desatualizado. Apesar destes dados, o fato de a paciente ter realizado o acompanhamento pré-natal associa-se significativamente à melhora na cobertura do exame citopatológico do colo uterino (IC95\% no início: 35,3-42,5 e IC95\% no final: 53,6-64,6) apresentando $\mathrm{p}<0,001$.

Conforme distribuição da amostra, observou-se que $80,2 \%$ das puérperas estudadas tinham idade igual ou inferior a 29 anos, $69,9 \%$ eram brancas e $91,2 \%$ apresentavam companheiro fixo. Apesar de 50,6\% das pacientes relatarem nove ou mais anos de escolaridade, 76,4\% referiram renda familiar per capita inferior a um salá- rio mínimo ( $\mathrm{R} \$ 380,00)$. O parto foi financiado pelo Sistema Único de Saúde (SUS) em 84,3\% dos casos (Tabela 1).

Em relação ao acompanhamento pré-natal da gravidez atual, 69,2\% das puérperas o realizaram pelo SUS (308), 48,5\% (216) em postos de saúde do município e 20,7\% (92) no Hospital Universitário (FURG). O restante das pacientes - $137(30,8 \%)$ - fez o pré-natal por algum tipo de convênio ou em consultórios particulares. Como este estudo foi realizado pelo método de amostragem, existe a possibilidade de viés de seleção. A idade gestacional média de ingresso no pré-natal foi de 12 semanas, sendo que 69\% das pacientes o iniciaram no primeiro trimestre. A média de consultas realizadas por gestante foi de 7,3 , verificando-se que $71 \%$ delas fizeram seis ou mais consultas. Quanto ao Índice de Kessner, $60,2 \%$ dos pré-natais foram classificados como adequados (Tabela 2).

Nos antecedentes obstétricos, verificou-se que $56,4 \%$ das mulheres referiam duas ou mais gestações e $51,2 \%$ eram multíparas. Quanto ao tabagismo, $68,1 \%$ das puérperas estudadas referiam nunca terem fumado. Em relação aos outros fatores de risco para o câncer do colo uterino, $85,8 \%$ das pacientes referiam início da vida sexual com idade inferior ou igual há 19 anos e $47,2 \%$ relatavam três ou mais parceiros sexuais durante suas vidas. Uma em cada duas puérperas $(51,2 \%)$ teve o primeiro parto com 19 anos ou menos (Tabela 3 ).

$\mathrm{Na}$ análise das variáveis demográficas e socioeconômicas, o grupo de puérperas com idade igual ou superior a 20 anos apresentou maior prevalência $(p=0,004)$ de CP nos últimos 36 meses, quando comparadas às mulheres de menor idade. As puérperas não brancas tiveram maior prevalência de citologia cervical desatualizada do que as pacientes brancas. Pacientes com escolaridade igual ou inferior a onze anos, pacientes com renda familiar per capita inferior a um salário mínimo e aquelas com parto financiado pelo SUS apresentaram maior risco significativo de terem menor cobertura do exame citopatológico no final do pré-natal (Tabela 1).

Levando em consideração as características do pré-natal na gravidez atual, encontrou-se maior probabilidade de não realização do exame de CP nos últimos três anos nas puérperas que fizeram o acompanhamento pré-natal no SUS (RP:1,86 e IC95\%:1,39-2,47). As mulheres com início do prénatal no $2^{\circ}$ e no $3^{\circ}$ trimestres de gravidez e que tiveram seu acompanhamento pré-natal classificado como inadequado pelo Índice de Kessner 
Tabela 1. Distribuição da amostra, análise da cobertura e de fatores demográficos e socioeconômicos associados à não atualização do exame citopatológico durante o pré-natal em município do Rio Grande do Sul, Brasil.

\begin{tabular}{|c|c|c|c|c|c|}
\hline Variável & $\mathbf{n}(\%)$ & $\begin{array}{c}\text { Sem } \\
\text { citopatológico }(\%)\end{array}$ & $\begin{array}{c}\text { Razão de } \\
\text { prevalência }\end{array}$ & $\mathrm{IC}_{95 \%}$ & p-valor \\
\hline Idade (anos) & & & & & 0,004 \\
\hline De 35 ou mais & $35(7,9)$ & $12(34,3)$ & 1,0 & & \\
\hline De 30 a 34 & $53(11,9)$ & $18(34,0)$ & 0,99 & $0,55-1,79$ & \\
\hline De 25 a 29 & $141(31,7)$ & $51(36,2)$ & 1,05 & $0,63-1,75$ & \\
\hline De 20 a 24 & $98(22,0)$ & $37(37,8)$ & 1,10 & $0,65-1,86$ & \\
\hline De 10 a 19 & $118(26,5)$ & $64(54,2)$ & 1,58 & $0,97-2,58$ & \\
\hline Cor da pele & & & & & 0,08 \\
\hline Branca & $311(69,9)$ & $119(38,3)$ & 1,0 & & \\
\hline Não branca & $134(30,1)$ & $63(47,0)$ & 1,23 & $0,98-1,54$ & \\
\hline Tem companheiro & & & & & 0,003 \\
\hline Sim & $406(91,2)$ & $157(38,7)$ & 1,0 & & \\
\hline Não & $39(8,8)$ & $25(64,1)$ & 1,66 & $1,27-2,16$ & \\
\hline Escolaridade (anos) & & & & & $<0,001$ \\
\hline 12 ou mais & $32(7,2)$ & $3(9,4)$ & 1,0 & & \\
\hline De 9 a 11 & $193(43,4)$ & $67(34,7)$ & 3,70 & $1,24-11,06$ & \\
\hline De 5 a 8 & $165(37,1)$ & $45(53,9)$ & 5,75 & $1,94-17,05$ & \\
\hline De 0 a 4 & $55(12,3)$ & $14(41,8)$ & 4,46 & $1,45-13,99$ & \\
\hline Renda familiar per capita & & & & & 0,001 \\
\hline Maior de 2 SM & $29(6,5)$ & $6(20,7)$ & 1,0 & & \\
\hline De 1,0 e $2,0 \mathrm{SM}$ & $76(17,1)$ & $23(30,3)$ & 1,46 & $0,66-3,22$ & \\
\hline Menor de 1,0 SM & $340(76,4)$ & $153(45,0)$ & 2,17 & $1,06-4,48$ & \\
\hline Financiamento do parto & & & & & 0,005 \\
\hline Particular ou convênio & $70(15,7)$ & $18(25,7)$ & 1,0 & & \\
\hline SUS & $375(84,3)$ & $164(43,7)$ & 1,70 & $1,12-2,57$ & \\
\hline
\end{tabular}

Obs.: renda familiar per capita: divisão da renda total da família pelo número de habitantes na casa. SM: salário mínimo $(\mathrm{R} \$ 380,00)$.

Tabela 2. Distribuição da amostra, análise da cobertura e características do pré-natal atual associadas à não atualização do exame citopatológico durante o pré-natal em município do Rio Grande do Sul, Brasil.

\begin{tabular}{|c|c|c|c|c|c|}
\hline Variável & n (\%) & $\begin{array}{c}\text { Sem } \\
\text { citopatológico }(\%)\end{array}$ & $\begin{array}{c}\text { Razão de } \\
\text { prevalência }\end{array}$ & $\mathrm{IC}_{95 \%}$ & p-valor \\
\hline Local do pré-natal & & & & & $<0,001$ \\
\hline Particular/convênio & $137(30,8)$ & $40(29,2)$ & 1,0 & & \\
\hline FURG & $92(20,7)$ & $25(27,2)$ & 0,93 & $0,61-1,42$ & \\
\hline SUS & $216(48,5)$ & $117(54,2)$ & 1,86 & $1,39-2,47$ & \\
\hline Início do pré-natal & & & & & 0,002 \\
\hline $1^{\mathrm{o}}$ trimestre & $307(69,0)$ & $110(35,8)$ & 1,0 & & \\
\hline $2^{\circ}$ ou $3^{\circ}$ trimestre & $138(31,0)$ & $72(52,2)$ & 1,46 & $1,17-1,81$ & \\
\hline Número de consultas & & & & & 0,08 \\
\hline 6 ou mais consultas & $316(71,0)$ & $121(38,3)$ & 1,0 & & \\
\hline De 1 a 5 consultas & $129(29,0)$ & $61(47,3)$ & 1,23 & $0,98-1,55$ & \\
\hline Índice de Kessner & & & & & 0,01 \\
\hline Adequado & $268(60,2)$ & $97(36,2)$ & 1,0 & & \\
\hline Inadequado & $177(39,8)$ & $85(48,0)$ & 1,33 & $1,06-1,65$ & 0,005 \\
\hline
\end{tabular}


Tabela 3. Distribuição da amostra, análise da cobertura e fatores de risco para câncer do colo uterino associados à não atualização do exame citopatológico durante o pré-natal em município do Rio Grande do Sul, Brasil.

\begin{tabular}{|c|c|c|c|c|c|}
\hline Variável & n (\%) & $\begin{array}{c}\text { Sem } \\
\text { citopatológico (\%) }\end{array}$ & $\begin{array}{c}\text { Razão de } \\
\text { prevalência }\end{array}$ & $\mathrm{IC}_{95 \%}$ & p-valor \\
\hline Número de gestações & & & & & 0,08 \\
\hline Uma gravidez & $194(43,6)$ & $92(47,4)$ & 1,0 & & \\
\hline De 2 a 3 gestações & $169(38,0)$ & $57(33,7)$ & 0,71 & $0,55-0,92$ & \\
\hline 4 ou mais gestações & $82(18,4)$ & $33(40,2)$ & 0,85 & $0,63-1,15$ & \\
\hline Número de partos & & & & & 0,13 \\
\hline Um parto & $217(48,8)$ & $102(47,0)$ & 1,0 & & \\
\hline De 2 a 3 partos & $165(37,1)$ & $52(31,5)$ & 0,67 & $0,51-0,87$ & \\
\hline 4 ou mais partos & $63(14,1)$ & $28(44,4)$ & 0,95 & $0,69-1,29$ & \\
\hline Fumo & & & & & 0,35 \\
\hline Não & $303(68,1)$ & $122(40,3)$ & 1,0 & & \\
\hline Ex-fumante & $58(13,0)$ & $22(37,9)$ & 0,94 & $0,66-1,35$ & \\
\hline Fumo atual & $84(18,9)$ & $38(45,2)$ & 1,12 & $0,86-1,48$ & \\
\hline Idade da primeira relação & & & & & $<0,001$ \\
\hline 20 anos ou mais & $63(14,2)$ & $17(27,0)$ & 1,0 & & \\
\hline 16 a 19 anos & $210(47,3)$ & $74(35,2)$ & 1,31 & $0,84-2,04$ & \\
\hline 15 anos ou menos & $171(38,5)$ & $91(53,2)$ & 1,97 & $1,28-3,03$ & \\
\hline Número de parceiros & & & & & 0,62 \\
\hline 1 a 2 parceiros & $235(52,8)$ & $99(42,1)$ & 1,0 & & \\
\hline 3 parceiros ou mais & $210(47,2)$ & $83(39,5)$ & 0,94 & $0,75-1,17$ & \\
\hline Idade do primeiro parto & & & & & $<0,001$ \\
\hline 30 anos ou mais & $40(9,0)$ & $10(25,0)$ & 1,0 & & \\
\hline 25 a 29 anos & $57(12,8)$ & $12(21,1)$ & 0,84 & $0,40-1,76$ & \\
\hline 20 a 24 anos & $120(27,0)$ & $42(35,0)$ & 1,40 & $0,78-2,52$ & \\
\hline 19 anos ou menos & $228(51,2)$ & $118(51,8)$ & 2,07 & $1,19-3,59$ & \\
\hline
\end{tabular}

apresentaram maior probabilidade, $46 \%$ e $33 \%$, respectivamente, de permanecerem com suas citologias cervicais desatualizadas (Tabela 2).

Em relação aos fatores de risco para o câncer do colo do útero, o grupo de mulheres com duas a três gestações e partos prévios apresentou menor probabilidade de estarem com o CP desatualizado no fim do pré-natal. As mulheres fumantes tiveram maior prevalência de CP desatualizado, embora essas variáveis não tenham demonstrado diferença estatística significante. Quanto ao início da vida sexual, as puérperas com sexarca aos 15 anos ou menos apresentaram risco 97\% maior de não terem realizado citologia cervical nos últimos três anos. A análise também mostrou diferença estatística significativa quanto à idade do primeiro parto $(\mathrm{p}<0,001)$. As mulheres que o tiveram com 19 anos ou menos apresentaram maior probabilidade (RP:2,07 e IC95\%:1,193,59 ) de permanecerem com o exame citopatológico desatualizado (Tabela 3 ).

A análise multivariada, incluindo todas as puérperas e ajustada para fatores de confusão, demonstrou que as variáveis estudadas não apresentaram associação estatística significativa para a não realização do exame citopatológico do colo uterino nos últimos três anos. É importante ressaltar a variável relacionada ao local de realização do pré-natal, em que se observa $\mathrm{p}=0,08$ com uma razão de prevalência ${ }^{1,18}$ (IC95\%:0,98-1,42) para as pacientes que realizaram suas consultas em postos de saúde do município credenciados no SUS.

\section{Discussão}

Segundo dados da literatura sobre causas de mortes evitáveis por ações efetivas dos serviços de saúde, o óbito por câncer do colo uterino é classificado como evitável pela prevenção primária em $30 \%$, pela prevenção secundária em $50 \%$ e pela prevenção terciária em $20 \%$. Ressalta-se que as avaliações das mortes por carcinoma cervical podem ser incluídas nas condições claramente usadas como indicadores da qualidade da assistência ${ }^{18}$. 
O presente estudo mostrou que 95,3\% das puérperas entrevistadas tinham conhecimento sobre o exame de prevenção do câncer do colo uterino. No entanto, 36\% das mulheres entrevistadas permaneceram sem nunca terem realizado a citologia cervical mesmo após o pré-natal. Apesar de ainda ter sido encontrado um grande número de mulheres, em Rio Grande, para as quais não foi oportunizada a prevenção do câncer cervical, este estudo mostrou uma melhora nos últimos dez anos. Um estudo de base populacional, realizado no mesmo município em 1995, incluindo 1.302 mulheres de 15 a 49 anos, que já haviam mantido relação sexual, encontrou uma prevalência de $57 \%$ de mulheres que nunca haviam realizado o exame de Papanicolaou ${ }^{19}$. Apesar desta aparente melhora na cobertura do CP, é importante ressaltar que as gestantes apresentam maior probabilidade de estarem com seu exame citopatológico atualizado em relação às mulheres não grávidas. Portanto, é provável que a cobertura de $\mathrm{CP}$ fora da gravidez no município do Rio Grande seja menor do que a demonstrada neste estudo.

Ainda em relação ao conhecimento e à cobertura do exame citopatológico, em uma pesquisa realizada no município de Teresópolis (RJ) foram realizadas entrevistas com 304 mulheres, que levaram seus filhos menores de um ano ao Dia Nacional de Vacinação. Observou-se que 25,3\% das mães referiram nunca terem realizado exame preventivo do câncer do colo de útero. Dentre as 227 mães que alguma vez realizaram o exame colpocitológico-oncótico, apenas $17,6 \%$ o fizeram durante o pré-natal ${ }^{14}$.

Estudo recente avaliou o conhecimento sobre pré-natal entre gestantes residentes na periferia da cidade do Rio Grande, observando que apenas $6,0 \%$ respondiam espontaneamente que o exame preventivo para o câncer do colo uterino fazia parte da rotina do pré-natal. Somente após o entrevistador perguntar sobre este exame é que $74,1 \%$ das pacientes passaram a incluí-lo no que acreditavam ser exame obrigatório no pré-natal. Mesmo assim, 19,9\% das gestantes responderam que o $\mathrm{CP}$ não fazia parte da rotina, mostrando o seu escasso conhecimento sobre os procedimentos do pré-natal. Os autores evidenciaram a necessidade de intensificar o processo educativo entre as gestantes, na tentativa de reduzir a assimetria na relação gestante-serviço de saúde e melhorar a qualidade da atenção ${ }^{20}$.

Segundo a OMS, o exame citopatológico do colo uterino deve alcançar uma cobertura em torno de $80 \%$ da população feminina entre 25 e 49 anos, para ter real efetividade na prevenção do carcinoma cervical ${ }^{1,5}$. Apesar desta orientação, quando se analisou a razão entre exames citopatológicos cérvico-vaginais em mulheres de 25 e 59 anos e a população feminina nesta faixa etária na cidade de Rio Grande, observou-se uma cobertura deste exame em 2006 de 6\% Ao realizar uma média dos últimos quatro anos, a razão de exames nesta cidade passa para 4\%, sendo a segunda pior cobertura de exame citopatológico do colo uterino do Estado $^{13}$. Estes valores são uma estimativa da cobertura do citopatológico em Rio Grande, e não as taxas reais. Além disso, não é esperado pelo Sistema de Informação do Câncer do Colo Uterino (Siscolo) uma cobertura maior que $30 \%$, pois o CP deve ser repetido trienalmente, e uma parcela significativa da população utiliza o sistema de saúde suplementar para a sua realização.

A prevalência de citologia cervical atualizada encontrada neste estudo foi de 39,9\% no início do pré-natal, chegando a 59,1\% no puerpério. Estudos que avaliem a cobertura do CP em gestantes no Brasil são raros. Na maioria das vezes, eles avaliam a assistência pré-natal e trazem referências à realização da citologia cervical. Uma avaliação da atenção pré-natal da rede básica realizada em Pelotas (RS) mostrou que em apenas $53,4 \%$ dos atendimentos havia o registro do CP, e destes apenas $35,6 \%$ encontravam-se atualiza$\operatorname{dos}^{16}$. No entanto, um estudo transversal com 702 gestantes cujo parto ocorreu no Hospital Geral da Universidade de Caxias do Sul (RS) mostrou uma taxa de coleta de citologia cervical no pré-natal de 51\%, sendo superior à encontrada nos estudos anteriores ${ }^{10}$.

$\mathrm{Na}$ análise bruta das características associadas à não atualização do exame citopatológico durante o pré-natal, podemos observar que as mulheres com idade entre 10 e 19 anos, que não apresentavam companheiro fixo, com renda familiar per capita menor que um salário mínimo e com escolaridade igual ou inferior a onze anos, apresentaram maior probabilidade de permanecerem com a sua citologia desatualizada no final do pré-natal. Revisão sistemática da literatura realizada em 2004, buscando informações sobre a cobertura do exame Papanicolau no Brasil, observou que os fatores que levam à não realização do exame de Papanicolaou têm se repetido nos diferentes estudos. Entre eles, estão: mulheres com baixo nível socioeconômico, baixa escolaridade e pertencentes às faixas etárias mais jovens ${ }^{21}$. Um estudo transversal de base populacional realizado em Pelotas (RS), que entrevistou 1.404 mulheres de 20 a 59 anos, mostrou diferença estatística 
significante associada à não realização do $\mathrm{CP}$ nos últimos três anos em relação às seguintes variáveis: faixa etária jovem, mulheres não brancas, escolaridade menor ou igual a quatro anos e não apresentarem companheiro fixo ${ }^{22}$.

A limitação do acesso aos serviços de saúde, por barreiras socioeconômicas, culturais e geográficas, também se apresenta como responsável pela baixa cobertura dos exames de citologia oncótica $^{23-25}$. No entanto, a cobertura pré-natal encontrada em nosso estudo foi de $96 \%$, sendo que $60,2 \%$ dos acompanhamentos foram considerados adequados pelo índice de Kessner. Mesmo assim, as pacientes que realizaram o acompanhamento da gestação nos postos de saúde do SUS apresentaram prevalência de citologia atualizada no fim do pré-natal de $45,8 \%$. Entre as pacientes que realizaram o pré-natal em consultórios privados ou na FURG, a prevalência de citologia atualizada foi de 70,8\% e 72,8\%, respectivamente. Alguns estudos ressaltam que mulheres com acesso a serviços credenciados e conveniados apresentaram maior probabilidade de serem submetidas ao exame citopatológico quando comparadas às mulheres que utilizam o sistema público ${ }^{23,24}$. Segundo Puccini et al. ${ }^{15}$, para gestantes com baixa renda, idade inferior a 20 anos e falta de acesso a plano privado de saúde, existe maior probabilidade de estas receberem assistência pré-natal de qualidade inferior.

Levando-se em consideração os fatores de risco relacionados ao câncer do colo uterino, esperava-se que as gestantes mais expostas fossem as mais examinadas. No entanto, observou-se que mesmo as mulheres com quatro ou mais gestações, fumantes ou ex-fumantes e com história de três ou mais parceiros sexuais não apresentaram maior prevalência de realização do exame citopatológico. Nos grupos que apresentaram diferença estatística significante, chama a atenção o das puérperas com a primeira relação aos 15 anos ou menos, pois apresentou uma prevalência de citologia atualizada de $46,8 \%$, valor este muito inferior à taxa encontrada entre as pacientes que relataram sexarca aos 20 anos ou mais $(73 \%)$, fato este também observado no grupo de pacientes que referiram o primeiro parto com idade igual ou inferior a 19 anos, em que a prevalência de citologia atualizada foi de 48,2\% em contraste com o grupo que teve seu primeiro parto aos 20 anos ou mais, que apresentou prevalência de $70,5 \%$ de citologia atualizada no final do pré-natal. Estudo de base populacional realizado em Rio Grande ${ }^{19}$ observou que o grupo de mulheres com a primeira relação aos 15 anos ou menos e com três ou mais filhos apresentaram uma frequência de realização da citologia cervical 20\% menor que a encontrada entre as mulheres com sexarca aos 20 anos ou mais e as nulíparas ou as secundigestas. Na literatura, outros estudos também encontraram menor cobertura do exame citopatológico do colo uterino entre as mulheres com maior risco para desenvolver este tipo de câncer ${ }^{21-24}$.

Quanto à prevalência da realização do citopatológico associado a fatores de barreira específicos, observa-se, após a análise ajustada, que a realização do pré-natal no Hospital Universitário da FURG encontra-se associada à maior prevalência de citologia cervical atualizada no puerpério, confirmando a teoria de que serviços ligados a universidades apresentam propedêutica mais bem estabelecida ${ }^{11,23,24}$. Outra variável que se mostrou individualmente associada à melhor cobertura do exame citopatológico foi a idade entre 20 e 24 anos.

Os resultados do presente estudo devem ser interpretados com cautela. Embora o número da amostra tenha sido adequado para estimar a prevalência do exame citopatológico do colo uterino, a amostra não foi calculada individualmente para os fatores associados à não realização deste exame. Outra limitação deste estudo diz respeito à validação de alguns dados colhidos por entrevistas, particularmente aqueles que dependem da memória e da boa vontade da informante. Há, neste ponto, alguma possibilidade de viés recordatório, visto que algumas mães podem ter esquecido ou omitido alguns dados durante a entrevista. Os possíveis aspectos de aferição poderiam ter sido minimizados pela avaliação do cartão do pré-natal. Apesar das limitações, os resultados deste estudo sugerem a viabilidade da metodologia utilizada, além de revelarem informações anteriormente desconhecidas, permitindo a identificação de deficiências importantes no processo de prestação de cuidados pré-natais e da prevenção do carcinoma cervical, na população de gestantes do município do Rio Grande.

Concluímos que, apesar de este estudo constatar um aumento da cobertura do exame citopatológico do colo uterino na cidade do Rio Grande nos últimos anos, o serviço local de saúde mostrou-se pouco efetivo e desigual, em se tratando da detecção precoce do carcinoma cervical neste município. Pouco efetivo porque cobriu menor número de mulheres do que o indicado pela $\mathrm{OMS}^{1}$, e desigual porque $\mathrm{o}$ acesso a esse exame variou conforme algumas características das usuárias. Sabendo que o rastreio precoce é o 
método mais importante para o controle do carcinoma cervical, torna-se mandatório estender este benefício a todas as mulheres, mas principalmente às mais vulneráveis, por meio de políticas e intervenções específicas que priorizem essa população. Essa medida poderia contribuir para redução da mortalidade por uma doença relativamente frequente e quase sempre evitável.

Assim, os resultados aqui apresentados revelam a necessidade de se aumentar a cobertura da citologia cervical e melhorar a qualidade da atenção pré-natal oferecida no município do Rio Grande. Ficou claro que é preciso motivar e capacitar os profissionais de saúde quanto à importância dos procedimentos da rotina pré-natal, especialmente o exame especular e a coleta do citopatológico. Segundo Cesar et al. ${ }^{19}, 90 \%$ das mulheres em idade fértil em Rio Grande fazem pelo menos uma consulta de pré-natal. Se somente nessa situação fosse realizado o exame citopatológico, seria possível diagnosticar cerca de metade dos casos de carcinoma cervical em estádio inicial, em que o percentual de cura é extremamente alto.

Torna-se imperativa a discussão desses resultados nos conselhos populares de saúde, no conselho municipal de saúde e com os programas que mais intensamente atingem a popula- ção de menor poder aquisitivo, em especial o Programa de Saúde da Família (PSF), o Programa de Agentes Comunitários de Saúde (Pacs) e a Pastoral da Criança, buscando ajudar a colocar em prática os princípios de universalidade e equidade na atenção da saúde da mulher, o que significa atender a todos de acordo com suas necessidades e obedecendo aos preceitos do SUS.

Por fim, gostaríamos de ressaltar que os estudos transversais - principalmente os baseados em amostras probabilísticas - sobre a cobertura do exame de Papanicolaou no Brasil e os fatores associados à sua não realização são poucos, e a maioria se encontra concentrada nas regiões Sul e no Sudeste do paíis ${ }^{21}$. Também seria importante aumentar o número de estudos que investigassem as práticas médicas básicas do pré-natal, que apesar de fazerem parte da rotina, parece que nem sempre são executadas. Como vemos no presente estudom o fato de ter um pré-natal avaliado como "adequado numericamente" não garante cuidados qualitativos adequados, visto que muitos profissionais da saúde deixam de aproveitar o momento da consulta pré-natal para realizar a coleta do exame citopatológico, perdendo essa oportunidade, talvez a única, de realizar a prevenção do câncer do colo uterino nessa mulher.

\section{Colaboradores}

CV Gonçalves, G Duarte, JSD Costa, SM Quintana e AC Marcolin participaram igualmente de todas as etapas de elaboração do artigo. 


\section{Referências}

1. World Health Organization (WHO). Comprehensive cervical cancer control: a guide to essential practice. Geneva: WHO; 2007.

2. Brasil. Ministério da Saúde. Instituto Nacional de Câncer (Inca). Normas e recomendações do Inca; prevenção e controle de câncer. Rev Bras Cancerol 2002; 48(3):317-332.

3. Brasil. Ministério da Saúde. Instituto Nacional de Câncer (Inca). Coordenação de Prevenção e Vigilância. Estimativa 2008: incidência de câncer no Brasil. Rio de Janeiro: Inca; 2007.

4. Deligdisch L, Miranda CRR, Wu HS, Gil J. Human papillomavirus-related cervical lesions in adolescents: a histologic and morphometric study. Gynecol Oncol 2003; 89(1):52-59.

5. Blumenthal PD, Gaffikin L. Cervical cancer prevention: making programs more appropriate and pragmatic. JAMA 2005; 294(17):2225-2228.

6. Van Calsteren K, Vergote I, Amant F. Cervical neoplasia during pregnancy: diagnosis, management and prognosis. Best Pract Res Clin Obstet Gynaecol 2005; 19(4):611-630.

7. Nygard M, Daltveit AK, Thoresen SO, Nygard JF. Effect of an antepartum Pap smear on the coverage of a cervical cancer screening programme: a population-based prospective study. BMC Health Services Research 2007; 7:10-18.

8. Jacobs JA, Chang CK, Salti GI. Coexistence of pregnancy and cancer. Am Surg 2004; 70(11):1025-1029.

9. Brasil. Ministério da Saúde. Pré-natal e puerpério: atenção qualificada e humanizada - manual técnico. Brasília: Ministério da Saúde; 2005.

10. Trevisan MR, Lorenzi DRS, Araújo NM, Ésber K. Perfil da assistência pré-natal entre usuárias do Sitema Único de Saúde em Caxias do Sul. Rev Bras Ginecol Obstet 2002; 24(5):293-299.

11. Neumann NA, Tanaka OU, Victora CG, César JÁ. Qualidade e equidade da atenção ao pré-natal e ao parto em Criciúma, Santa Catarina, Sul do Brasil. Rev Bras Epidemiol 2003; 6(4):307-318.

12. Koffman MD, Bonadio IC. Avaliação da atenção pré-natal em uma instituição filantrópica da cidade de São Paulo. Rev Bras Saúde Matern Infant 2005; 5(1):23-32.

13. Instituto Brasileiro de Geografia e Estatística (IBGE). Censos demográficos e contagem populacional para os anos intercensitários, estimativas preliminares dos totais populacionais, estratificadas por idade e sexo pelo MS/SE/Datasus. [acessado 2008 jan 28]. Disponível em: http://www.datasus.gov.br

14. Santos SR, Cunha AJL, Gamba CM, Machado FG, Leal JMMF, Moreira NLM. Avaliação da assistência à saúde da mulher e da criança em localidade urbana da região Sudeste do Brasil. Rev Saude Publica 2000; 34(3):266-271.

15. Puccini RF, Pedroso GC, Silva EMK, Araújo NS, Silva NN. Eqüidade na atenção pré-natal e ao parto em área da Região Metropolitana de São Paulo, 1996. Cad Saude Publica 2003; 19(1):35-45.
16. Silveira DS, Dias da Costa JS, Santos IS. Atenção prénatal na rede básica: uma avaliação da estrutura e do processo. Cad Saude Publica 2001; 17(1):131-139.

17. Victora CG, Huttly SR, Fuchs SC, Olinto MTA. The role of conceptual frameworks in epidemiological analysis: a hierarchial approach. Int J Epidemiol 1997; 26(1):224-247.

18. Malta DC, Duarte EC. Causas de mortes evitáveis por ações efetivas dos serviços de saúde: uma revisão da literatura. Cien Saude Colet 2007; 12(3):765776.

19. Cesar JA, Horta BL, Gomes G, Holthausen RS, Willrich RM, Kaercher A, Iastrenski FM. Fatores associados à não realização de exame citopatológico de colo uterino no extreme Sul do Brasil. Cad Saude Publica 2003; 19(5):1365-1372.

20. Mendonza-Sassi RA, César JÁ, Ulmi EF, Mano OS, Dall'Agnol MM, Neumann NA. Avaliando o conhecimento sobre pré-natal e situações de risco à gravidez entre gestantes residentes na periferia da cidade de Rio Grande, Rio Grande do Sul, Brasil. Cad Saude Publica 2007; 23(9):2157-2166.

21. Martins LFL, Thuler LCS, Valente JG. Cobertura do exame de Papanicolaou no Brasil e seus fatores determinantes: uma revisão sistemática da literatura. Rev Bras Ginecol Obstet 2005; 27(8):485-492.

22. Hackenhaar AA, Cesar JA, Domingues MR. Exame citopatológico de colo uterino em mulheres com idade entre 20 e 59 anos em Pelotas, RS: prevalência, foco e fatores associados à sua não realização. Rev Bras Epidemiol 2006; 9(1):103-111.

23. Dias da Costa JS, Olinto MTA, Gigante DP, Menezes AMB, Macedo S, Borba AT, Motta GLS, Fuchs SC. Cobertura do exame citopatológico na cidade de Pelotas, Rio Grande do Sul, Brasil. Cad Saude Publica 2003; 19(1):191-197.

24. Novaes HMD, Braga PE, Schout D. Fatores associados à realização de exames preventivos para câncer nas mulheres brasileiras, PNAD 2003. Cien Saude Colet 2006; 11(4):1023-1035.

25. Agurto I, Bishop A, Sanchez G, Betancout Z, Robles S. Perceived barriers and benefits to cervical cancer screening in Latin America. Prev Med 2004; 39(1):91-98.

Artigo apresentado em 17/08/2009

Aprovado em 25/09/2009

Versão final apresentada em 30/10/2009 